LANGE, J.-M. (2017). Éducation au développement durable: enjeux épistémologiques et didactiques des reconfigurations disciplinaires possibles. In Ch. VergnolleMainar (dir.) Curricula et disciplines scolaires : changements et reconfigurations (pp. 181-191). Toulouse: Presse Universitaire du Midi.

\title{
Education au développement durable : enjeux épistémologiques et didactiques des reconfigurations disciplinaires possibles.
}

\author{
Jean-Marc Lange \\ Laboratoire CIVIIC (EA 2657) \\ Jean-marc.lange@univ-rouen.fr
}

\section{Résumé}

$\mathrm{Si}$, par cohérence avec les recommandations et prescriptions internationales et nationales, l'éducation au développement durable (EDD) prend la forme d'un curriculum piloté par des actions éducatives participatives, la question d'un complément de celles-ci par une contribution des disciplines scolaires (Lange et Martinand, 2010) se pose à la fois dans un souci d'acceptabilité de l'EDD pour les acteurs scolaires en France et dans celui de l'évitement du piège de l'indifférenciation des savoirs ou de l'anomie (Martinez, 2011).

Pour autant plusieurs modalités possibles de contribution disciplinaire coexistent comme ont pu l'établir les études menés dans le cadre de la recherche "Education au développement durable : appuis et obstacles ». Les changements occasionnés par l'introduction de l'EDD dans les curricula peuvent prendre la forme d'une recherche de convergence, de complémentarité, voire de reconfiguration autour de concepts organisateurs, celle d'une réélaboration des contenus avec une orientation épistémologique et éducative transformée, ou encore une centration sur le cœur des disciplines contributives.

Mots-Clés : Education au Développement Durable - Curriculum - Reconfiguration des disciplines scolaires -Contribution disciplinaires

Keywords: Education for sustainable development - Curriculum - Reconfiguration of school subjects Contribution of school subjects 
Définir le rôle de l'École dans l'élaboration d'un développement humain sous condition de durabilité est un enjeu fort qui dépasse largement le seul projet de sensibilisation trop fréquemment évoqué à propos de l'éducation au développement durable (EDD). La question posée au travers de l'idée de DD est celle des modalités permettant l'émergence d'un «vivre ensemble » renouvelé. La place de l'Ecole est centrale dans cette affaire car comme l'affirme Gaucher (cité par Forquin, 2003, p 118) :

\section{L'école est aujourd'hui l'institution où le problème principal de la démocratie, en tant que régime des droits de l'individu, à savoir le problème de l'articulation de l'individu avec le collectif, est testé avec le plus d'acuité.}

Pour cet auteur, le rôle de l'éducation institutionnelle est celui d'une École pensée comme laboratoire de nouveaux rapports individu/collectif. De même, Rosanvallon (2011) pose le constat d'un fossé qui se creuse entre une citoyenneté politique et une citoyenneté sociale, du fait d'un repli individualiste ou communautaire et identitaire. Ce fossé conduit progressivement au déchirement de la démocratie. L'École, pensée comme institution au service de la société et comme fondement de la démocratie, offre alors l'opportunité de le combler. Mais les enjeux actuels ne peuvent nous amener seulement à poser le problème de l'altérité, même si cette question est essentielle, car les enjeux environnementaux se posent également de façon cruciale. Nous prolongeons donc la proposition précédente par celle d'une École pensée comme laboratoire, lieu d'innovation et d'expérimentation, de nouveaux rapports Nature/Sciences/Société.

Ce projet éducatif est donc bien politique mais le projet d'une éducation institutionnelle ne l'a-t-il pas toujours été ? La nouveauté de la situation est que ce projet prend forme dans un contexte de crise de la représentativité démocratique qui engendre un sentiment croissant de défiance vis-à-vis des élites et des experts (Rosanvallon, 2006). La question de l'acceptabilité d'une forme curriculaire de l'EDD pour les acteurs premiers de l'école, c'est-à-dire les enseignants, les élèves et les familles, est donc un enjeu crucial car soumis au risque de son rejet 
par les familles et les élèves, détournement ou rabattement par les concepteurs des programmes et/ou les enseignants ${ }^{1}$.

Par ailleurs, la forme curriculaire qui s'est imposée pour le second degré au cours du $\mathrm{XX}^{\mathrm{e}}$ siècle est celle d'une structuration en disciplines conçues, pour une part, comme transposition des disciplines académiques de référence. Le rapport entre «éducation à un développement durable » et disciplines scolaires est bien une question vive pour les parties-prenantes de la scolarité obligatoire.

\section{Quel type curriculaire acceptable pour les acteurs d'une éducation au développement durable scolaire?}

Le politiste Ross (2001) a analysé les variations historiques des conceptions curriculaires, les positions politiques, les présupposés idéologiques, les tensions, les implications éducatives et pédagogiques, relatifs à l'élaboration du «national curriculum » anglais. Dans une perspective beaucoup plus générale, ce chercheur a proposé une analyse politique des curriculums en termes de grands modes ou types de pilotage : soit par des contenus, souvent organisés en disciplines ou en matières, soit par des objectifs, en particulier aujourd'hui des objectifs de compétences, soit par l'intervention sur un processus d'activités pour en exploiter les potentialités éducatives. Ross souligne les incompatibilités existant entre ces types curriculaires pris deux à deux, les impossibilités de chacun, et la forte teneur idéologique des préférences pour ces diverses manières de conduire un curriculum.

Reprenant à notre compte les distinctions de Ross, nous avons proposé une centration sur des actions participatives, partenariales, négociées et territoriales de développement durable. Ayant retenu la stratégie de la pratique sociale comme modalité éducative pour l'EDD, par prise au sérieux des textes de préconisations (notamment Brégeon, 2008), se pose alors la question des conditions de possibilité d'un tel curriculum. La proposition d'un complément de cette stratégie par des investigations portant sur les enjeux de durabilité et celui de disciplines scolaires pensé en termes de contribution à l'EDD, a été effectuée (Lange et Martinand, 2010 ; Lange, 2011 ; Lange, 2014).

1 Cette question a fait l'objet d'une partie des travaux menés dans le cadre de la recherche ANR «ED2AO » [2009-2012, ANR-08-BLAN-0135-01]; 


\title{
Diversité et limites des modes de contributions possibles
}

\author{
Reconfigurations disciplinaires internes par entrée de contenus \\ nouveaux, intérêts et limites
}

Partant du principe que les contenus notionnels et conceptuels disciplinaires peuvent être des appuis pour l'EDD, mais au prix de leur réélaboration dans une perspective d'étayage de la pensée, plusieurs études de didactique ont porté sur des analyses critiques des programmes existants et ou sur des travaux à caractère prospectif. Les travaux rapportés ici sont notamment relatifs aux disciplines scolaires de l'enseignement général et obligatoire, mais également aux curriculums de l'enseignement agricole. Ils sont de nature macrocurriculaire (Lebeaume, 2012) dans leur méthodologie.

Ainsi, la question de la biodiversité a-t-elle été récemment introduite dans les programmes de Sciences de la Vie et de la Terre (SVT). Les études de didactique menées à ce jour (Barroca-Paccard, Orange-Ravachol et Gouyon, 2013) établissent d'une part qu'il s'agit davantage d'une coloration thématique que d'une construction curriculaire cohérente et assumée du fait de l'existence de lacunes conceptuelles centrales (diversité génétique des espèces ...) ou périphériques (potentiel évolutif des espèces ...), associées à une coloration seulement patrimoniale et biologique de la question.

Dans ce même domaine disciplinaire, à propos de la question de la production alimentaire, l'analyse des textes de prescriptions que nous avons effectuée (Lange, 2014) converge avec la précédente: des lacunes centrales (système agro-alimentaire ; agro-écologie ; ressources halieutiques...) et périphériques (services écosystémiques ...) apparaissent, et la teneur générale des programmes est productiviste et « techno-chimico-génétique » centrée.

Dans une visée prospective, Ducamp et Simonneaux (2010) ont étudié quant à elles les conditions de possibilité de l'introduction de la chimie verte et ses douze principes dans les programmes de lycée de section agricole. Dans cette recherche, ont été recueillies les représentations d'enseignants sur le développement durable, l'environnement et la chimie verte. D'une part, les enseignants de l'enseignement agricole associent la chimie verte à la chimie produite 
à partir de plantes. D'autre part, leurs représentations font écho aux représentations sociales négatives de la chimie le plus souvent mises en avant dans les discours médiatisés. Le risque d'une posture de défense de la chimie s'associe donc à une méconnaissance de ces développements récents chez les enseignants ayant fait l'objet de cette enquête. La question de l'acceptation et de l'appropriation par les enseignants de ces approches nouvelles, associant contenus nouveaux et pratiques renouvelées, se pose alors.

Reconfigurations disciplinaires internes par recherche des spécificités, apports et limites

En nous appuyant sur l'exemple de la biodiversité et celui de risque, nous avons pu élaborer une typologie de la contribution du domaine disciplinaire SVT à l'éducation au développement durable (Lange, 2011), cherchant ainsi à prolonger et adapter à ce nouveau contexte ce que Martinand (1996) a nommé le «cœur»des disciplines : «cœur actuel » des disciplines académiques, «cœur potentiel » des disciplines scolaires. Trois contributions majeures nous semblent ainsi possible.

Comme étayage pour l'action en faveur du DD (ex: biodiversité)

L'un des défis sociétaux clés de la stratégie nationale du DD, est celui de l'évolution de la biodiversité. Celle-ci est devenue, au travers d'indices internationaux, un des paramètres de la mesure de l'état de «santé » d'une planète placée sous «monitoring », et par voie de conséquence, un enjeu et un indicateur de la durabilité ou de la nondurabilité des actions et modalités effectives de développement.

Pour l'enseignant de sciences, la mission est triple :

- permettre l'appropriation et l'usage de l'idée en tant qu'indicateur, par exemple en participant à des programmes nationaux de recensement tel que le propose notamment le Muséum National d'Histoire Naturelle (MNHN) en France (visée dispositionnelle),

- permettre la compréhension de sa signification de l'idée audelà du catastrophisme trop rapide et à sa réduction à la seule diversité des espèces, ou d'espèces emblématiques et médiatisée (visée épistémologique), 
- mais aussi révéler la part de dérive idéologique (espèces invasives ...) et de scientisme (réduction de la question de la gestion de la biodiversité à sa seule dimension biologique) potentiels que l'idée de biodiversité peut trop facilement suggérer (visée éthique).

Comme entrée dans les controverses scientifiques par l'appropriation des démarches modélisantes (ex: empreinte écologique)

Le concept de capacité de charge prend ses sources dans les travaux portant sur les dynamiques de population en écologie au tournant du $19^{\mathrm{e}}$ et du $20^{\mathrm{e}}$. Sa filiation remonte aux travaux de Malthus à la fin du $18^{\mathrm{e}}$. Exprimé en population limite et mathématisé en 1838 sous la forme d'un modèle, la loi logistique, par le mathématicien belge Verhulst, le concept est «redécouvert» en 1927 par le généticien New-Yorkais Pearl. C'est cette idée, mais inversée, qui est globalisée à l'ensemble planétaire dans le cadre de l'empreinte écologique.

Par ses travaux, Pearl (1927) entendait montrer que la croissance des populations humaines répond à des facteurs biologiques fondamentaux qui permettent de négliger les facteurs économiques et sociaux. C'est donc un projet de naturalisation des questions de société qui était promu dans un cadre de pensée relevant lui-même largement d'une idéologie scientiste.

Ce qui importe du point de vue épistémologique et éducatif, audelà de la valeur descriptive, explicative, prédictive ou d'aide à la conceptualisation d'un modèle, ce sont ses limites et les incertitudes qui en résultent, mais aussi la capacité qu'il offre pour évaluer une situation, à la comparer à d'autres : sa fonction d'aide à la décision. Du point de vue de l'EDD, cette fonction peut être fondatrice d'une citoyenneté active.

Usage, appropriation et recours à des démarches modélisantes diversifiées, et reconnaissance de leurs limites, sont le cœur de l'enseignement des SVT et fondent une modalité de sa contribution aux questions de société.

Comme compréhension partielle d'une question de société (ex: risque et vulnérabilité)

Sur le plan sociétal, il est maintenant admis que deux registres d'appréhension du risque se confrontent : registre scientifique, le plus 
souvent exprimée en termes de probabilités, et registre profane, trop souvent qualifié d'irrationnel par les experts et décideurs, dépendant de l'appartenance territoriale et du sentiment de vulnérabilité liée au risque considéré. Ces deux registres définissent l'acceptabilité du risque (Faucheux et Nicolaï, 2005).

Socialement, cette affaire est d'importance car elle conditionne la tenue du débat démocratique. Ainsi, Chevassus-au-Louis (2007) précise la distinction apportée : l'appréciation du risque par les experts est effectuée par le biais de ses attributs quantitatifs et probabilistes et ce dans une rationalité scientifique. Tout autre est l'appréciation du risque par les citoyens : ceux-ci envisagent le risque par ses attributs qualitatifs selon une rationalité profane, qualitative et tournée vers soi, qu'il ne faut pas confondre avec l'irrationalité, et posent son caractère juste ou injuste.

La contribution des sciences de la nature se limite à la compréhension de l'aléa et de sa probabilité de survenue. C'est dans cette compréhension de l'aléa et l'appréciation rationnelle et hiérarchisée du risque qui en résulte que réside la contribution de l'éducation scientifique à l'EDD à cette question. Mais la compréhension globale et complexe de la vulnérabilité nécessite une approche multiréférentielle mêlant une fois de plus sciences de la nature et sciences humaines et sociales, et laissant de la place à une rationalité profane.

Réduire l'étude du risque à sa seule dimension «sciences de la nature » peut être le vecteur d'une conception technocratique du monde par naturalisation de la question, au détriment du débat démocratique fondé sur la multiplicité des points de vue et donc des possibles (Fourez, 1994). La décision démocratique, quant à elle, repose sur la reconnaissance de la complémentarité des appréciations du risque dont il est question.

\section{Reconfiguration interdisciplinaire du point de vue des contenus}

Partant d'une analyse curriculaire de l'existant, Vergnolle Mainar (2011) met en évidence, au moyen du calcul du poids horaire relatif des domaines disciplinaires, un retournement de leur hiérarchie dans la prise en charge de l'EDD : SVT et géographie, de périphériques deviennent centrales dans le système des disciplines. Il s'agit là d'une première reconfiguration essentielle, résultant probablement des jeux 
d'influence des lobbies disciplinaires dans la recherche de la légitimation de leurs domaines.

Du point de vue des contenus, et ce au moyen d'une démarche d'analyse historique de ces mêmes disciplines, Vergnolle Mainar distingue des concepts nomades (écosystèmes, paysage aménagé ...) et des concepts organisateurs émergents (pour la géographie : milieu, espace, territoires). Mais ces changements ne se traduisent pas par des changements en profondeurs des curriculums ni du point de vue épistémologique, ni du point de vue didactique : ces disciplines restent dans leur logique interne traditionnelles et très positivistes. Les rencontres interdisciplinaires restent marginales, d'autant que les textes de programmes sont peu diserts sur ce point, malgré les préconisations du socle commun.

Dans la perspective d'une interdisciplinarité, Vergnolle Mainar, suggère de rechercher des convergences fortes. C'est ainsi qu'elle identifie des concepts devenus fédérateurs, tels ceux de paysage ou de ressources, ce que confortent nos propres analyses relatives à la question de la production alimentaire en SVT et géographie, du moins sous la forme d'ilots multiréférentiels (Lange, 2014).

\section{Pour une approche curriculaire renouvelée}

Globalement, il apparait une certaine convergence entre les analyses de ces didacticiens sous la forme de la mise en évidence de tensions potentielles existant entre légitimité académique et légitimité sociale, entre savoirs scientifiques et savoirs profanes, entre savoirs académiques et savoirs de l'ingénieur, entre savoirs du management et savoirs académiques (Corpus étudié ; Barthes, Zwang et Alpe, 2013). Ces tensions seraient alors déterminantes dans l'acceptabilité des contenus de l'EDD et de leur introduction dans les curriculums existants.

Selon Tutiaux-Guillon (2011), la recherche des complémentarités interdisciplinaires risque d'une part d'entrainer un effet d'indifférenciation, ou de confusion épistémologique, des disciplines centrales de l'EDD. La mise en évidence du risque d'indifférenciation rejoint les analyses effectuées par Martinez (2010) du point de vue de la philosophie de l'éducation. Pour cette auteure, le risque majeur résultant de l'introduction scolaire de l'éducation au développement durable, est celui d'une anomie - l'incapacité à nommer et par là 
même à penser le monde - résultant du processus d'indifférenciation des ordres du savoir, notamment les disciplines, renforçant ainsi celui de l'indifférenciation des cultures issue de la mondialisation néolibérale.

D'autre part, toujours pour Tutiaux-Guillon, l'affichage des complémentarités, dans la volonté de leur mise en relation, pourrait se faire au détriment de celle de la recherche d'une problématisation spécifique pour chacune d'entre elles. Pour cette auteure la dérive ainsi soulignée prend sa source dans la prégnance d'un paradigme positiviste qui légitime à l'Ecole les savoirs disciplinaires et disqualifie les savoirs vernaculaires ou d'expérience.

Vergnolle Mainar (2011) souligne enfin la valorisation des noyaux durs des disciplines comme étant l'un des obstacles majeurs à la mise en synergie interdisciplinaire. Dépassant de fait l'idée de concept fédérateur, cette auteure reprend alors à son compte l'idée d' "ilots interdisciplinaires de rationalité » développée par Fourez (1997) comme moyen de transformer en profondeur les curriculums existants mais surtout leur mise en œuvre. La question de la réalité des pratiques interdisciplinaires et celle des conditions institutionnelles, pédagogiques et didactiques dans le second degré sont en effet fondamentales et devraient faire l'objet d'un travail de mise à jour par la recherche en éducation.

Si la perspective ainsi élaborée rejoint finalement notre idée de compléter les actions participatives et négociées de développement durable par des «investigations multiréférentielles», elle s'en distingue par l'importance relative accordée aux savoirs académiques et aux savoirs profanes, y compris domestiques, aux croyances et idéologies à l'œuvre. Cette question constitue le nœud de tension, le cœur de la balise curriculaire à prendre en compte pour un dépassement pourtant nécessaire de l'opposition existant entre partisans d'une recherche de convergence et partisans d'une recherche des spécificités disciplinaires, entre partisans de modes de pilotages curriculaires concurrents.

Pour échapper au risque d'indifférenciation, ou pour dépasser l'obstacle de naturalisation des questions de sociétés, nous posons celles-ci comme investigations portant sur les enjeux d'un développement humain sous contrainte de durabilité. Seule, selon nous, cette démarche curriculaire, conservant le cœur et la spécificité des disciplines, et leur potentiel formateur, permettra de passer du 
difficile dialogue interdisciplinaire à une reconstruction transdisciplinaire (Martinand, 2008) opérationnelle pour la formation citoyenne par la mise à jour des enjeux sociétaux et leur élucidation, l'étayage de l'opinion par les savoirs et méthodes scientifiques, la reconnaissance de leurs apports et limites, la confrontation des points de vues et la déconstruction des discours qui s'y rapportent, l'apprentissage de la décision au nom du bien commun et celui des négociations qu'elle nécessite.

C'est la question des finalités et des moyens de l'EDD scolaire qui se trouve ainsi posée : s'agit-il d'éclairer le citoyen en l'instruisant des résultats de la science ou de permettre une problématisation et la recherche collective de solutions nouvelles et plurielles, au moyen notamment d'actions éducatives et du maintien d'un processus de formation par et aux disciplines ?

\section{Bibliographie}

BARTHes Angela, Zwang Aurélie, Alpes Yves, Sous la bannière développement durable, quels rapports aux savoirs scientifiques? Revue Education Relative à l'Environnement - Regards-Recherche Réflexion, 11, 2013, p. 81-98;

BREGEON Jacques, Rapport du groupe de travail interministériel sur l'Éducation au développement durable, Ministère de l'Éducation Nationale, Paris, 2008 ;

Chevassus-au-Louis Bernard L'analyse des risques, l'expert, le décideur et le citoyen, Quae, Versailles, 2007 ;

FAUCHEUX Sylvie, NicOlaÏ Isabelle, Environnement et société : de l'évaluation du risque à son acceptation, Sociétal 50, 2005, p. 26-32 ;

FORQUIN Jean-Claude, La critique communautarienne du libéralisme politique et ses implications possibles pour l'éducation, Revue française de pédagogie 143, 2003, p. 113-139.

FORQUIN, Jean-Claude, Organisation des savoirs, dans VAN ZANTEN Agnès (dir.) Dictionnaire de l'éducation, PUF, Paris, 2008 ;

FOUREZ Gérard, Qu'entendre par « Îlot de rationalité »? Et par « Îlot interdisciplinaire de rationalité »? Aster 25, 1997, p. 217-225;

FOUREZ Gérard, Constructivisme et justification éthique, Revue des sciences de l'éducation 20, 1, 1994, p. 157-174, http://id.erudit.org/iderudit/031705ar 
LANGE Jean-Marc, Éducation au développement durable : intérêts et limites d'un usage scolaire des investigations multiréférentielles d'enjeux, dans Fabre Michel, Hagege Hélène, Reynaud Christian Les éducations à ... et le développement de la pensée critique, Education et socialisation, les cahiers du CERFEE 36, 2014, consultable à http://edso.revues.org/872.

LANGE Jean-Marc, Education au Développement Durable:problématique éducative / problèmes de didactique, mémoire d'Habilitation à Diriger des Recherches, ENS Cachan, 2011 ;

LANGE Jean-Marc, MARTINAND Jean-Louis, Education au développement durable et éducation scientifique : Repères pour un curriculum, dans HASNI Abdelkarim, LEBEAUME Joël (dir) «Enjeux contemporains de l'éducation scientifique et technique », Les Presses de l'Université d'Ottawa, Canada, 2010 ;

LEBEAUME Joël, Effervescence contemporaine des propositions d'éducations à... Regard rétrospectif pour le tournant curriculaire à venir, Spirale-Revue de Recherches en Education, 50, 2012, p. 1124 ;

MARTINAND Jean-Louis, Environnement et interdisciplinarité : paradoxes dans le champ éducatif, EDP Sciences/Natures Sciences Sociétés, 16, 2008, p. 1-2;

MARTINAND Jean-Louis, D'où est venue la didactique ?, Éducations, 7, 1996, p. 22-25 ;

MARTINEZ Marie-Louise, Approche anthropologique de l'éducation à l'écoresponsabilité et l'écocitoyenneté, dans MUSTIERE Philippe, FABRE Michel (dir.), Les rencontres Jules Vernes "Science, Technique et Société : de quoi sommes-nous responsables?», Coiffard librairie éditeur, Nantes, 2010 ;

Rosanvallon Pierre, La contre-démocratie, Seuil, Paris, 2006 ;

RosanVALLON Pierre, La société des égaux, Seuil, Paris, 2011 ;

Ross Allistair, Curriculum : construction and Critique, RoutledgeFalmer, London and New York, 2000 ;

\section{Sources du corpus étudié}

Borroca-PACCARD Marco, Orange-Ravachol Denise, GouYon Pierre-Henri, Education au développement durable et diversité du vivant : la notion de biodiversité dans les programmes des sciences de 
la vie et de la Terre, Penser l'éducation, Hors série, 2013, p. 467484 ;

DuCAMP Christine, SimonneAuX Laurence, Chimie verte: qu'en savent les enseignants de l'Enseignement Agricole dans la perspective de l'EDD ? dans AlPe Yves, GiRAult Yves, Actes du Colloque «Education au développement durable et à la biodiversité » IUT de Provence, Digne les Bains, Réseau Francophone International de la recherche en Education relative à l'environnement, Université du Québec à Montréal, www.refere.uquam.ca, 2010 ;

LANGE Jean-Marc, La question de la production alimentaire et de sa durabilité dans les prescriptions de l'enseignement général : analyse curriculaire, Revue Francophone du développement durable, Les questions socialement vives, 4, 2014, p. 142-154;

LANGE Jean-Marc, Penser en termes de contribution l'éducation scientifique à l'éducation au développement durable, Revue des Hautes Ecoles Pédagogiques 13, 2011, p. 137-156 ;

TUTIAUX-GUILlON Nicole, Histoire-géographie et éducation au développement durable en France : tensions et redéfinitions, dans BADER Barbaber, SAUVE Lucie (dir.), Education, environnement et développement durable:vers une écocitoyenneté critique, Québec : Presses de l'Université Laval, 2011, p.125-160;

VERGNOLLE-MAINAR Christine, La géographie dans l'enseignement, une discipline en dialogue, Presses universitaires de Rennes, Rennes, 2011. 
